\title{
Résumé des recommandations pour la prévention de la contraction d'hépatites virales en voyage
}

\author{
Comité consultatif de la médecine tropicale et de la médecine des voyages (CCMTMV)*
}

* Auteur-ressource : catmat.secretariat@phac-aspc.gc.ca

\section{Résumé}

Les hépatites virales sont considérées comme les maladies les plus courantes liées au voyage et évitables par la vaccination. Tous les voyageurs non immuns qui se rendent dans des pays en développement devraient envisager la vaccination en recevant un vaccin inactivé contre l'hépatite $A$ et un vaccin contre l'hépatite $B$ recombiné. Le vaccin inactivé contre l'hépatite $A$ et le vaccin contre l'hépatite $B$ recombiné sont sécuritaires, entraînent peu d'effets secondaires et fournissent une protection à long terme. Tous les vaccins monovalents contre l'hépatite $A$ et l'hépatite $B$ offerts au Canada ont la même efficacité et peuvent être administrés de manière interchangeable. Les immunoglobulines de l'hépatite A ne devraient être utilisées pour prévenir l'hépatite A que chez les personnes pour qui les vaccins contre l'hépatite A sont contre-indiqués, chez les personnes immunodéprimées qui pourraient ne pas réagir correctement aux vaccins actifs ou chez les nourrissons de moins d'un an. Tous les voyageurs devraient adopter des mesures de protection de routine à l'étranger. Les porteurs du virus de l'hépatite $B$ qui voyagent dans un pays où l'hépatite $D$ est endémique devraient être particulièrement vigilants et éviter les activités à risque élevé, comme le perçage de la peau et les pratiques sexuelles non sécuritaires.

\section{Contexte}

L'hépatite A est la maladie liée au voyage la plus courante pour laquelle il y a un vaccin efficace. Une carte des pays et des régions à risque d'infection par les virus de l'hépatite $A$ et de l'hépatite $B$ est disponible auprès de l'Organisation mondiale de la Santé. Des renseignements détaillés sur les vaccins contre l'hépatite $A$ et I'hépatite B se trouvent dans le Guide canadien d'immunisation : épidémiologie au Canada, vaccins offerts, recommandations d'utilisation au Canada, précautions, contre-indications, etc. Un résumé complet des facteurs de risques de l'hépatite $A$ et de l'hépatite $B$ par les voyageurs et des recommandations se trouvent dans la Déclaration sur les vaccins contre l'hépatite à l'intention des voyageurs du Comité consultatif de la médecine tropicale et de la médecine des voyages (1).

\section{Prévention}

\section{Recommandations en matière de vaccins pour les voyageurs canadiens}

Le vaccin inactivé contre l'hépatite $A$ et le vaccin contre l'hépatite $B$ recombiné sont sécuritaires, entraînent peu d'effets secondaires et fournissent une protection à long terme $(2,3)$. On considère qu'ils sont tous deux sans danger durant la grossesse et l'allaitement $(3,4)$. Tous les vaccins monovalents contre l'hépatite A et l'hépatite B offerts au Canada ont la même efficacité et peuvent être administrés de manière interchangeable pour lutter contre leur virus ciblé respectif $(3,5)$.

Tous les voyageurs non immuns qui se rendent dans des pays en développement devraient envisager la vaccination en recevant un vaccin inactivé contre l'hépatite $A$ et un vaccin contre l'hépatite B recombiné (2, 6-9).

L'administration du vaccin contre l'hépatite A est considérée comme efficace jusqu'au jour du départ et ne requiert pas l'administration d'immunoglobulines $(10,11)$. 
II est recommandé que les voyageurs qui n'ont pas d'antécédents de vaccination contre l'hépatite B, ou pour lesquels l'antécédents est incomplète, de recevoir une série vaccinale avant leur départ. Si des voyageurs ne peuvent recevoir la série vaccinale complète avant leur départ, l'administration d'une ou deux doses de vaccin contre l'hépatite $B$ avant le voyage pourrait fournir tout de même une certaine protection et constituera le début d'une série vaccinale qui pourra être terminée au retour (1).

Dans le cas des voyageurs qui consultent moins de 21 jours avant le départ, il y a lieu d'administrer séparément les vaccins monovalents contre l'hépatite $A$ et l'hépatite $B$, et le reste des deux séries vaccinales au retour (1).

Les voyageurs atteints de l'hépatite $C$ devraient recevoir les vaccins contre l'hépatite $A$ et l'hépatite $B$, s'ils n'ont pas déjà été administrés par leur fournisseur de soins primaires (1).

Le vaccin contre l'hépatite $A$, le vaccin contre l'hépatite $B$ et le vaccin combiné hépatite $A$ /hépatite $B$ peuvent être administrés en même temps que d'autres vaccins, mais à un point d'injection différent et à l'aide d'une aiguille et d'une seringue distinctes (3).

\section{Immunoglobulines}

Le vaccin est l'agent de prophylaxie préexposition à privilégier pour l'hépatite A. Ainsi, les immunoglobulines ne devraient être utilisées pour prévenir l'hépatite $A$ que chez les personnes pour qui les vaccins contre l'hépatite $A$ sont contre-indiqués, chez les personnes immunodéprimées qui pourraient ne pas réagir correctement aux vaccins actifs ou chez les nourrissons de moins d'un an $(1,3)$.

L'administration concomitante d'immunoglobulines et du vaccin contre l'hépatite A n'est pas nécessaire (à l'exception peut-être des personnes immunodéprimées) et peut entraîner une réponse immunitaire moindre au vaccin contre l'hépatite $A(10,12,13)$.

II n'existe pas de préparation d'immunoglobulines ou de vaccin efficace disponibles au Canada qui offrent une protection contre les virus des hépatites $C$, D et $E(9,14-18)$.

II peut être raisonnable de supposer l'immunité des personnes qui ont de fortes chances d'avoir déjà été exposées et pour qui le coût de l'immunisation est un problème important (p. ex. personnes ayant longtemps vécu dans une région où la maladie est endémique ou personnes ayant des antécédents de maladie compatibles). Si le temps le permet avant le voyage, il peut être financièrement avantageux de tester la présence d'immunoglobulines $G$ dirigées contre le virus de l'hépatite $A$ chez les adultes qui ont grandi dans un pays en développement ou qui sont nés au Canada avant 1945 et d'immuniser les personnes à risque seulement $(3,19$, 20).

\section{Test sérologique de routine}

II n'est pas nécessaire de procéder à des tests sérologiques après la vaccination contre l'hépatite A ou l'hépatite $B$, sauf chez les travailleurs de la santé et les voyageurs immunodéprimés. La séroprotection de ces personnes devrait être confirmée $(3,21,22)$. Le Guide canadien d'immunisation recense les groupes à risque pour lesquels un test sérologique de l'hépatite B est recommandé avant l'immunisation.

II n'est pas nécessaire de procéder à des tests sérologiques de routine pour déterminer la présence d'immunoglobulines $G$ dirigées contre le virus de l'hépatite $B$ (anticorps dirigé contre l'antigène de surface de l'hépatite B) chez les voyageurs déjà immunisés, à l'exception des travailleurs de la santé chez qui la présence de ces anticorps n'a jamais été vérifiée (3).

\section{Mesures de protection individuelle}

II faut conseiller aux voyageurs de prendre les mesures suivantes : 
- Suivre de près les recommandations relatives à l'eau et aux aliments et se laver les mains fréquemment, afin de réduire au minimum les possibilités d'exposition aux virus de l'hépatite $A$ ou de l'hépatite $E$ (23-25).

- Adopter des pratiques sexuelles plus sécuritaires et éviter tout perçage de la peau volontaire, comme le tatouage, l'acupuncture ou le perçage corporel, afin de réduire au minimum les possibilités d'exposition aux virus de l'hépatite $B$, de l'hépatite $C$ ou de l'hépatite $D$.

- Faire très attention au moment d'obtenir des soins médicaux ou dentaires nécessitant une intervention percutanée ou effractive, afin de réduire au minimum les possibilités d'exposition aux virus de l'hépatite $B$, de l'hépatite $C$ ou de l'hépatite $D(1,21)$.

Les porteurs du virus de l'hépatite $B$ qui voyagent dans un pays où l'hépatite $D$ est endémique devraient être particulièrement vigilants et éviter les activités à risque élevé, comme le perçage de la peau et les pratiques sexuelles non sécuritaires (1).

L'immunoglobuline humaine n'offre pas une protection contre les virus de l'hépatite $C$, de l'hépatite $D$ ou de I'hépatite $E$, et, à l'heure actuelle, aucun vaccin ciblant ces infections n'est disponible. 
Références

(1) Comité consultatif de la médecine tropicale et la médecine des voyages. Déclaration sur les vaccins contre l'hépatite à l'intention des voyageurs. Relevé des maladies transmissibles au Canada 2008; 34(DCC-2):1-24.

(2) Keystone JS. Travel-related hepatitis B: Risk factors and prevention using an accelerated vaccination schedule. Am J Med. 2005; 118(10):63-68.

(3) Agence de la santé publique du Canada. Guide canadien d'immunisation. 2014. Accès : http://www.phac-aspc.gc.ca/publicat/cig-gci/index-fra.php. [consulté le 29 mai 2014].

(4) Duff B, Duff P. Hepatitis A vaccine: Ready for prime time. Obstet Gynecol. 1998; 91(3):468-471.

(5) Zuckerman JN, Kirkpatrick CT, Huang M. Immunogenicity and reactogenicity of Avaxim (160 AU) as compared with Havrix (1440 EL.U) as a booster following primary immunization with Havrix (1440 EL.U) against hepatitis A. J Travel Med. Mars 1998; 5(1):18-22.

(6) Zuckerman JN, Steffen R. Risks of hepatitis B in travelers as compared to immunization status. J Travel Med. 2000; 7(4):170-174.

(7) Mutsch M, Spicher VM, Gut C, Steffen R. Hepatitis A virus infections in travelers, 1988-2004. Clin Infect Dis. 15 février 2006; 42(4):490-497.

(8) Van Damme P, Van Herck K. Effect of hepatitis A vaccination programs. JAMA. 13 juillet 2005; 294(2):246-248.

(9) Centers for Disease Control and Prevention. CDC Health Information for International Travel 2014. New York: Oxford University Press; 2014.

(10) Connor BA. Hepatitis A vaccine in the last-minute traveler. Am J Med. Octobre 2005; 118:58-62.

(11) Sagliocca L, Amoroso P, Stroffolini T, Adamo B, Tosti ME, Lettieri G, et al. Efficacy of hepatitis A vaccine in prevention of secondary hepatitis A infection: A randomised trial. Lancet. 3 avril 1999; 353(9159):1136-1139.

(12) Werzberger A, Mensch B, Kuter B, Brown L, Lewis J, Sitrin R, et al. A controlled trial of a formalininactivated hepatitis A vaccine in healthy children. N Engl J Med. 13 août 1992; 327(7):453-457.

(13) Bader TF. Hepatitis A vaccine. Am J Gastroenterol. Février 1996; 91(2):217-222.

(14) Heymann DL. Viral Hepatitis C. In: Control of Communicable Disease Manual. 19e éd. Washington, DC: American Public Health Association; 2008. p. 293-295.

(15) Heymann DL. Viral Hepatitis E. In: Control of Communicable Diseases Manual. 19 éd. Washington, DC: American Public Health Association; 2008. p. 298-300.

(16) Khuroo MS. Viral hepatitis in international travellers: Risks and prevention. Int J Antimicrob Agents. Février 2003; 21(2):143-152.

(17) Puro V, De Carli G, Scognamiglio P, Porcasi R, Ippolito G, Studio Italiano Rischio Occupazionale HIV. Risk of HIV and other blood-borne infections in the cardiac setting: Patient-to-provider and provider-to-patient transmission. Ann NY Acad Sci. Novembre 2001; 946:291-309.

(18) Heymann DL. Delta Hepatitis. In: Control of Communicable Diseases Manual. $19^{\mathrm{e}}$ éd. Washington, DC: American Public Health Association; 2008. p. 295-297. 
(19) Grabenstein J. Hepatitis A vaccine. ImmunoFacts. 2006:175-85.

(20) Ford, P.M., White, C., Kaufmann, H., MacTavish, J., Pearson, M., Ford, S., et al. Séroprévalence de I'hépatite $c$ dans un pénitencier fédéral pour femmes au canada. Relevé des maladies transmissibles au Canada. 1995; 21(14):132-134.

(21) Hutin YJ, Hauri AM, Armstrong GL. Use of injections in healthcare settings worldwide 2000: Literature review and regional estimates. BMJ. 8 novembre 2003; 327:1075-80.

(22) Pasricha N, Datta U, Chawla Y, Singh S, Arora SK, Sud A, et al. Poor responses to recombinant HBV vaccination in patients with HIV infection. Trop Gastroenterol. Octobre-décembre 2005; 26(4):178182.

(23) Comité consultatif de la médecine tropicale et la médecine des voyages. Déclaration sur la diarrhée du voyageur. Relevé des maladies transmissibles au Canada. 2001; 27:1-12.

(24) Organisation mondiale de la Santé. Hépatite A. 2013.

Accès : http://www.who.int/mediacentre/factsheets/fs328/fr/ [consulté le 28 mai 2014].

(25) Organisation mondiale de la Santé. Hépatite E. 2013.

Accès : http://www.who.int/mediacentre/factsheets/fs280/fr/ [consulté le 28 mai 2014].

\section{Remerciements}

Ce résumé a été préparé et révisé par Teitelbaum P., Libman M., Crockett M., McCarthy A. et Geduld J. (Agence de la santé publique du Canada).

Membres du CCMTMV : Boggild A., Brophy J., Bui Y. G., Crockett M., Ghesquiere W., Greenaway C., Henteleff A., Libman M., Teitelbaum P. et McCarthy A. (présidente)

Représentants chargés de la liaison : Hui C. (Société canadienne de pédiatrie) et Gershman M. (Centers for Disease Control and Prevention [États-Unis]).

Membres d'office : Marion D. (Centre des services de santé des Forces canadiennes, ministère de la Défense nationale), McDonald P. (Division des médicaments anti-infectieux, Santé Canada), Schofield S. (Direction de la protection de la santé de la Force, ministère de la Défense nationale) et Tepper M. (Direction de la protection de la santé de la Force, ministère de la Défense nationale).

Membre émérite : Jeanes C. W. L.

\section{Conflit d'intérêts}

Aucun déclaré 